\title{
Reanimación hídrica: fisiología de la respuesta a volumen
}

\author{
Luis A. Gorordo-Delsol1,2*, José O. Montoya-Rojo ${ }^{3}$, Graciela Merinos-Sánchez \\ Marcos A. Amezcua-Gutiérrez ${ }^{1}$ y Guillermo D. Hernández-López ${ }^{1,5}$ \\ ${ }^{1}$ Unidad de Cuidados Intensivos Adultos, Hospital Juárez de México; ${ }^{2}$ Unidad de Cuidados Intensivos, Unidad Médica de Alta Especialidad \\ Dr. Victorio de la Fuente Narváez, IMSS; ${ }^{3}$ Unidad de Cuidados Intensivos, Hospital Regional de Especialidades N. ${ }^{\circ} 30$, IMSS; ${ }^{4}$ Servicio de Urgencias \\ Adultos, Hospital General de México Dr. Eduardo Liceaga; ${ }^{5}$ Unidad de Cuidados Especiales Posquirúrgicos, Unidad Médica de Alta Especialidad \\ Dr. Victorio de la Fuente Narváez, IMSS. Ciudad de México, México
}

\section{Resumen}

La reanimación hídrica es un tratamiento farmacológico fundamental en la mayoría de los pacientes. Requiere un profundo entendimiento de la fisiología cardiovascular para identificar los mecanismos adaptativos y patológicos que rodean a la fluidoterapia. Este manuscrito reúne definiciones teóricas y conceptos operativos sobre los elementos miocárdicos y vasculares que se involucran en la respuesta a volumen; se analizan los componentes de la precarga, el retorno venoso y la contractibilidad, para finalmente esbozar la poscarga, en el complejo ciclo de fenómenos mecánicos, eléctricos y bioquímicos que hacen del flujo sanguíneo óptimo una meta de nuestros tratamientos.

Palabras clave: Terapia hídrica. Reanimación. Fisiología.

\section{Fluid resuscitation: physiology of volumen responsiveness}

\section{Abstract}

Fluid resuscitation is a fundamental pharmacological treatment in most patients. It requires a deep understanding of cardiovascular physiology in order to identify the adaptive and pathological mechanisms that surround the fluid therapy. This manuscript brings together theoretical definitions and operative concepts on the myocardial and vascular elements involved in the volume response; the components of preload, venous return and contractility are analyzed to finally outline the afterload in the complex cycle of mechanical, electrical and biochemical phenomena that make optimal blood flow a goal of our treatments.

Key words: Fluid therapy. Resuscitation. Physiology.

\section{Introducción}

La terapia hídrica es una de las principales herramientas de la medicina de todos los tiempos, es difícil encontrar a un paciente hospitalizado que no tenga un acceso vascular permeable con una solución «de base». Además, la fluidoterapia es piedra angular de la reanimación de los pacientes críticamente enfermos. Sin embargo, evaluar la capacidad de un organismo (léase corazón, circulación pulmonar y sistémica, endotelio, etc.) para tolerar una determinada cantidad de soluciones y generar una respuesta

\section{Correspondencia:}

*Luis A. Gorordo-Delsol

E-mail: luis.gorordodelsol@icloud.com
Disponible en internet: 03-12-2020
Fecha de recepción: 03-05-2019

Fecha de aceptación: 18-10-2020 DOI: $10.24875 / R E I E .19000039$
Rev Educ Investig Emer. 2019;1(4):145-151
www.medicinadeemergencias.com

Rev Educ Investig Emer. 2019;1(4):145-151
www.medicinadeemergencias.com México SA de CV. Este es un artículo open access bajo la licencia CC BY-NC-ND (http://creativecommons.org/licenses/by-nc-nd/4.0/). 
favorable requiere un profundo entendimiento de la fisiología de la cinética de los líquidos; estos principios y mecanismos se engloban en un concepto: respuesta a volumen. El presente artículo es el segundo de una serie sobre reanimación hídrica. En el artículo previo ${ }^{1}$ se trataron los tipos de líquidos y eventos adversos, aquí se profundiza en la respuesta fisiológica a la administración de líquidos, mientras que los autores planean un artículo adicional sobre la evaluación en la práctica diaria en urgencias.

Se define como respuesta a volumen la capacidad miocárdica de incrementar el gasto cardiaco (GC) más del $15 \%$ sobre el valor inicial, como resultado de una intervención diagnóstica o diagnosticoterapéutica ${ }^{2}$, aunque múltiples autores aceptan un GC > 10\% sobre el inicial ${ }^{3-9} 0$ un incremento del índice cardiaco (IC) $>10 \%{ }^{3}$, valor que no queda claro cuándo y cómo fue establecido, pues los retos de líquidos originales de Weill y Shoemaker determinaron mejoría clínica y cambios en el volumen sanguíneo (VS) y la presión venosa central $(P V C)^{10-12}$, mas nunca GC.

\section{Precarga, presión y volumen}

La precarga es la cantidad de tensión sobre una cavidad muscular (p. ej., aurícula, ventrículo) que existe en el momento justo antes de su contracción ${ }^{13}$, en este contexto se refiere a la presión telediastólica (PTD). La precarga es el resultante de varias fuerzas, sin embargo, no todas tienen un papel significativo en función de la respuesta a volumen, por lo que esta revisión se limita al análisis de los componentes más relevantes. Matemáticamente se expresa como: (presión telediastólica $\mathrm{x}$ radio telediastólico)/(2 $\mathrm{x}$ grosor de la pared).

En pacientes sanos, durante el reposo el VS es constante. Se puede estimar mediante la fórmula de Baker ${ }^{14}$, donde VS $=2.68$ I x área de superficie corporal (SC), por ejemplo, un varón de $70 \mathrm{~kg}$ de peso y talla de $1.7 \mathrm{~m}$ tiene una SC de $1.81 \mathrm{~m}^{2}$, por la constante de Baker resulta en $4.85 \mathrm{I}^{15}$; aproximadamente el $70 \%$ del VS se encuentra en el sistema venoso, este volumen se divide en dos: el volumen venoso estresado (25 a $30 \%)$ y no estresado $(70 \text { a } 75 \%)^{16}$.

EI VS venoso no estresado se considera volumen de reserva, pues no tiene efectos en el flujo sanguíneo. Su función es mantener distendidas a las venas, sin generar tensión sobre la pared (similar a la capacidad residual funcional pulmonar), pero mantiene el espacio endovascular abierto. El volumen estresado es el que generará flujo, se puede entender mediante un modelo estático (es decir, de flujo $0 \mathrm{ml} / \mathrm{min}$ ), donde se puede creer que un solo latido expulsará un determinado volumen sistólico que, al ser eyectado del ventrículo, generará una ola de sangre por el sistema arterial (una onda de pulso). Esta ola pierde su energía cinética cuando atraviesa el capilar arterial y entra al territorio venoso, donde la distensibilidad de las paredes vasculares será los suficientemente permisiva para incrementar el volumen venoso transitoriamente, es decir, al volumen no estresado preexistente se agregará más volumen proveniente de la circulación arterial; este volumen añadido produce tensión sobre la pared venosa mientras que la pared intenta contraerse en un retroceso elástico, y ya que las arterias son menos distensibles ${ }^{17,18}$ el flujo de sangre generado por el retroceso elástico de la vena será anterógrado. Otro concepto necesario es el de capacitancia, que se define como el volumen total contenido, es decir la suma del volumen estresado y no estresado ${ }^{16}$; el cambio en la capacitancia venosa ocurre cuando el tono simpático se incrementa y hay constricción venosa, por lo que parte del volumen no estresado pasará a ser volumen estresado y producirá tensión sobre la pared vascular y entonces aumento del flujo venoso. Un reflejo vasoconstrictor puede incrementar de 10 a $20 \mathrm{ml} / \mathrm{kg}$ el volumen estresado ${ }^{19,20}$.

Revisados los conceptos de volumen estresado y no estresado, distensibilidad venosa y capacitancia venosa, se puede integrar otro componente importante de la precarga: la presión media de llenado circulatorio (MCFP, mean circulatory filling pressure), parte elemental del modelo de Guyton descrita previamente por Weber $^{21}$, que representa el promedio de todas las presiones de toda la circulación durante el flujo sanguíneo, provocada por el retroceso elástico de todas las paredes vasculares sobre el total del volumen circulatorio; entonces la MCFP se incrementa con una carga de líquidos, vasopresores con efecto venoso o disminuyendo la presión de la aurícula derecha $\left(\mathrm{P}_{\mathrm{RA}}\right)$ (aumentando el volumen estresado, disminuyendo la distensibilidad venosa, al disminuir la presión yuxtacardiaca, que se explica más adelante), y por lo tanto disminuye con vasodilatadores y durante la hipovolemia (incrementando la capacitancia) o al aumentar la $\mathrm{P}_{\mathrm{RA}}$ por cualquier mecanismo. La presión media de llenado sistémico (MSFP, mean systemic filling pressure) es el promedio de todas las presiones intravasculares provocada por el retroceso elástico sobre el volumen circulatorio, pero a diferencia de la MCFP, la MSFP se obtiene durante ausencia de flujo22. La MCFP es la que provoca el flujo de sangre hacia la aurícula derecha, pues se contrapone a la resistencia venosa $\left(R_{v}\right)$ y a la $P_{R A}$, el flujo de sangre que llega al corazón (léase retorno venoso [VR]), que 
es básicamente la diferencia MCFP $-\mathrm{P}_{\mathrm{RA}}{ }^{23}$, donde no está clara la relevancia de la $R_{v}{ }^{24}$, y se expresa $V R=$ $\left(\right.$ MCFP-P $\left.{ }_{\text {RA }}\right) / R_{v}$ (Fig. 1).

En relación a la $\mathrm{P}_{\mathrm{RA}}$, los trabajos clásicos de fisiología explican cómo es un determinante del $\mathrm{VR}^{13,22-24}$, mientras que otros han cuestionado este dogma proponiendo que la $P_{R A}$ es dependiente del VR y otras presiones ${ }^{27}$, debido a que la posición anatómica de la aurícula la expone a otras presiones fuera del sistema circulatorio, como las presiones ejercidas sobre el pulmón por la ventilación mecánica, la presión intraabdominal y su mecanotransducción a la caja torácica, la presión de la aurícula opuesta y ambos ventrículos, la apertura valvular, el contenido entre la pleuras, del mediastino y el saco pericárdico, e incluso arritmias y las alteraciones de relajación miocárdica durante la diástole, que en condiciones normales se compensa y fluctúa poco, pero en condiciones patológicas puede tener una repercusión tan grave como el colapso de las cavidades derechas, aumento de la resistencia del flujo venoso, tamponade cardiaco y más. A estas presiones externas se las conoce como presión yuxtacardiaca; la suma de estas presiones de forma "concéntrica» sobre la aurícula derecha ocasionan modificaciones de esta y no necesariamente están relacionadas con el adecuado VR, volumen circulante o $G^{28,29}$. Medir la $P_{R A}$ resulta técnicamente complejo, por lo que, desde hace más de un siglo, se ha utilizado la PVC como un subrogado aceptable ${ }^{29,30}$, siempre y cuando se comprenda que la PVC no refleja exclusivamente la fuerza generada por el flujo venoso contra las paredes de la aurícula, sino todas las presiones ejercidas sobre la aurícula derecha.

EI VR tiene un límite y puede presentarse en múltiples patologías, por ejemplo en hipovolemia, donde el volumen estresado es insuficiente para generar tensión sobre las paredes de las venas y estas se colapsan, a esto se llama limitante de flujo, pues a pesar de que la $P_{R A}$ puede bajar hasta $0 \mathrm{mmHg}$, la $R_{v}$ que se opone al flujo no puede ser abolida, el VR está entonces limitado por la capacidad del volumen estresado a generar una MSFP mayor a la $P_{R A}$ y vencer a la $R_{V}$, lo que se expresa como VR $=M S F P / R_{v}{ }^{16,17,31}$, estos pacientes están secos, pálidos y con otros datos de hipoperfusión debido a la hipovolemia; en el lado opuesto, cuando la $\mathrm{P}_{\mathrm{RA}}$ está muy elevada por aumento de la presión yuxtacardiaca (estenosis valvular, tamponade, presiones del ventilador elevadas, etc.), donde incluso con $R_{v}$ baja, la MSFP no logra superar a la $P_{R A}$, lo que disminuye el flujo sanguíneo hacia el corazón, esto tendrá un impacto negativo sobre el GC que se explica

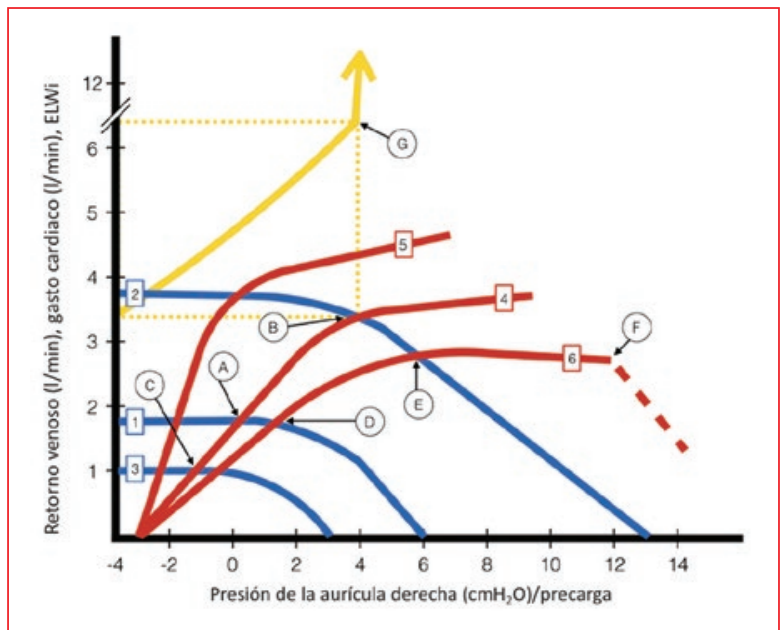

Figura 1. Curvas de relación entre la presión y el flujo/ volumen. Se lee la MCFP (azul) de derecha a izquierda, donde a menor $\mathrm{P}_{\mathrm{RA}}$ mayor es el flujo del retorno venoso, cuando la MCFP es normal (curva 1), cuando la MCFP está aumentada (curva 2), cuando la MCFP está disminuida (curva 3). La función ventricular (rojo), de izquierda a derecha, representa el aumento del gasto cardiaco (infiriendo frecuencia cardiaca estable) en función del aumento de la precarga de forma asintótica, es decir, primero de forma ascendente, donde por cada aumento de la precarga hay aumento del GC y posteriormente de forma plana, donde a pesar del aumento de la precarga no hay aumento del GC; la función ventricular normal (curva 4), cuando aumenta la contractibilidad ventricular, aumenta la distensibilidad diastólica o disminuye la poscarga (curva 5) o en presencia de insuficiencia cardiaca (curva 6). El punto $A$ señala que cuando la $P_{R A}$ es cercana a $0 \mathrm{cmH}_{2} \mathrm{O}$ y la MCFP es normal, si se administra líquido la MCFP aumenta hasta el punto $B$, lo que genera aumento del GC; si se pierde volumen sanguíneo o hay vasodilatación con una adecuada compensación contráctil, se llega al punto $C$; el punto $D$ señala la intersección entre la función cardiaca disminuida, con MCFP normal y $P_{\text {RA }}$ aumentada. $\mathrm{Si}$ a estos pacientes se les agrega volumen o venoconstrictores, la MCFP y la $P_{\text {RA }}$ aumentan sustancialmente, mientras que el GC solo aumenta poco (punto E); si esta tendencia continúa, la función cardiaca puede disminuir, sin impacto o con disminución de la MCFP y con aumento importante de la $P_{\text {RA }}$ (punto F) Finalmente, el ELWi muestra que mientras la función cardiaca (curva 4) se encuentra en la parte ascendente, el ELWi se mantiene dentro de su rango normal, y se eleva abruptamente (punto G), cuando se pierde la respuesta a volumen (punto B) esta línea no tiene correlación con la presión auricular (elaboración de los autores basada en Marik, et al.2014; ; Henderson, et al., 201022; Guyton, $1995^{25}$ y Sarnoff, 195426).

MCFP: presión media de llenado circulatorio, mean circulatory filling pressure; GC: gastocardiaco; $\mathrm{P}_{\mathrm{RA}}$ : presión de la aurícula derecha; ELWi: índice de agua extravascular pulmonar. 

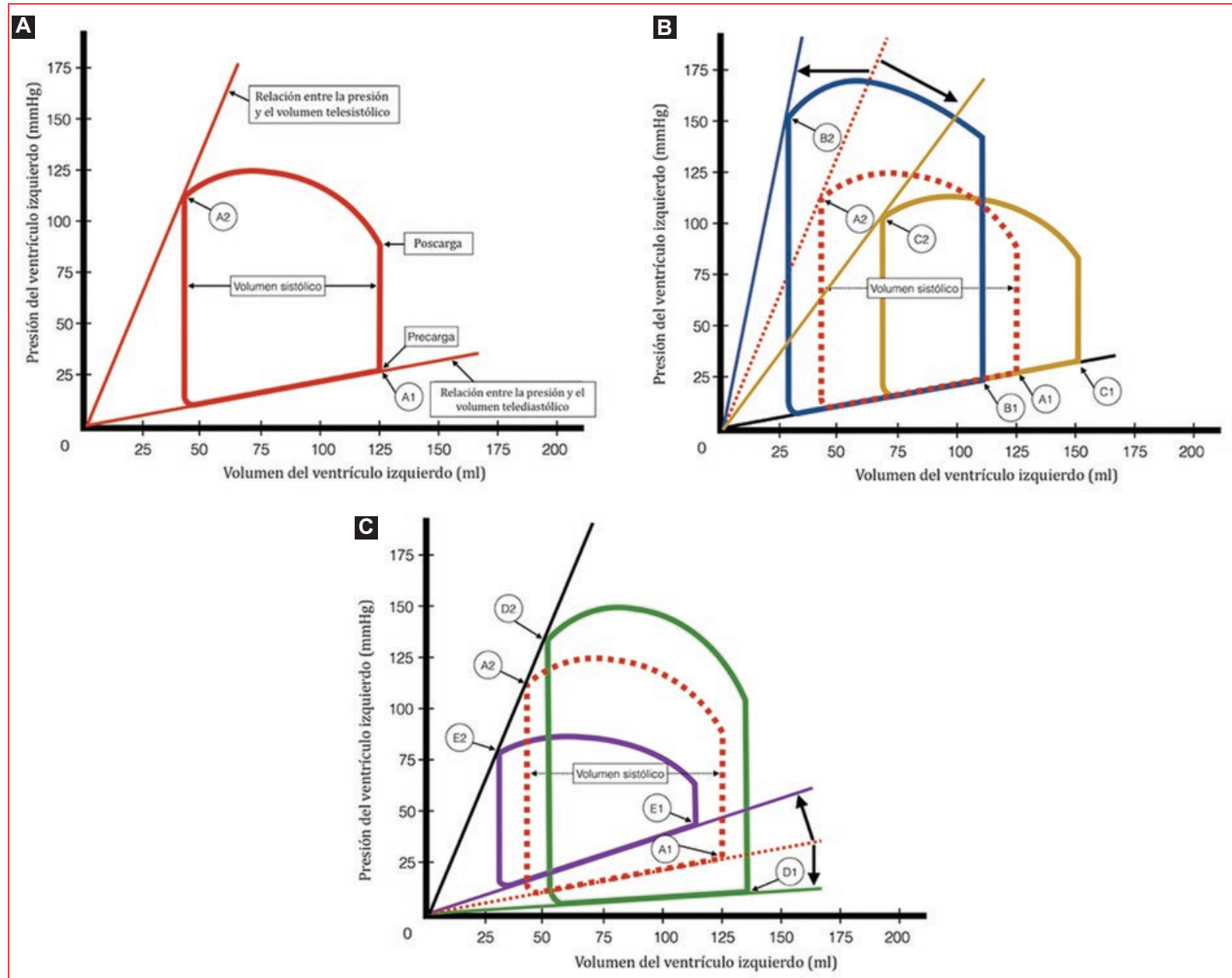

Figura 2. Curvas esquemáticas de función ventricular, donde se esquematiza la relación entre la presión y volumen del ventrículo izquierdo. A: se observa la función normal y se señalan el punto A1 el inicio de la sístole, unión entre la PTD y el VTD, mientras que A2 representa el fin de la sístole, unión entre la PTS y el VTS. B: muestra el aumento (azul) y disminución (amarillo) del inotropismo, el punto B1 indica que la sístole inicia con menos PTD y VTD, mientras que el punto B2 se desplaza arriba y a la izquierda para terminar con mayor PTS y VTS, el punto C1 indica que la sístole inicia con más PTD y VTD, mientras que el punto C2 se desplaza abajo y a la derecha para terminar con menor PTS y VTS. C: se muestra el aumento (verde) y disminución (violeta) del lusitropismo, el punto D1 indica que la sístole inicia con menos PTD y más VTD, mientras que el punto D2 se desplaza arriba y a la derecha para terminar con mayor PTS y VTS, el punto E1 indica que la sístole inicia con más PTD y menos VTD, mientras que el punto E2 se desplaza abajo y a la izquierda para terminar con menor PTS y VTS; de esta forma todas las variantes mantienen un volumen sistólico constante (elaboración de los autores basada en Guyton, et al., 200613 y Katz, 198834).

PTD: presión telediastólica; VTD: volumen telediastólico; PTS: presión telesistólica; VTS: volumen telesistólico.

a más adelante $16,17,20,25,32$. A estos pacientes se les observa ingurgitados, disneicos y edematosos debido al aumento de la presión hidrostática retrógrada.

\section{Ley del corazón}

Dentro de los alcances de esta revisión no se contemplan los trastornos de conducción (y el impacto del cronotropismo, el batmotropismo y el dromotropismo sobre el GC y la respuesta a volumen), sino los efectos del VR sobre el miocardio.

La capacidad del miocardio para relajarse (lusitropismo) es fundamental para el VR, pues da lugar al periodo de relajación isovolumétrica y posteriormente la fase de llenado que concluye al final de la diástole (con la presión y volumen telediastólico [VTD]), dicha relajación no 
es un proceso uniforme debido a la diferente velocidad con la que se desenrollan las fibras miocárdicas durante la relajación, la torsión de las fibras miocárdicas fomenta que se almacene energía potencial que será liberada en la contracción; como el miocardio se compone de múltiples capas de músculo en fibras helicoidales entretejidas, durante el llenado de las cavidades, la extensión de las fibras aumenta y su orientación se modifica tanto en el eje longitudinal como transmura ${ }^{33,34}$. Se explica entonces que el aumento del lusitropismo (léase más facilidad de relajación) modifica la curva de función ventricular para tolerar mayor VTD con menor PTD, lo que puede lograrse con algunos fármacos, mientras que la disminución del lusitropismo tiene el efecto contrario, como en miocardiopatía restrictiva, enfermedades infiltrativas y miocardiopatía hipertrófica ${ }^{34}$ (Fig. 2).

Comprender la importancia de una adecuada diástole permite vislumbrar cómo iniciará la sístole. La «ley del corazón», descrita por Starling, se basó en los resultados de sus modelos experimentales, demostrando que «lo que sale del ventrículo es lo mismo que regresa a la aurícula derecha» ${ }^{32}$. El mecanismo de Frank-Starling descrito a partir de estos experimentos fue reportado de una forma inusual, lo que ha ocasionado errores en la comprensión de la curva, pues se graficó la $P_{R A}$ como variable independiente y al GC como dependiente, en lugar del VR como la variable independiente (eje de las abscisas « $\mathrm{X}$ ) y la $\mathrm{P}_{\mathrm{RA}}$ y $\mathrm{GC}$ como dependientes (eje de las ordenadas «y») ${ }^{32}$. Posteriormente Sarnoff, et al. describieron la correlación entre la presión auricular con el volumen latido y el trabajo ventricular de cada lado del corazón en modelos animales sanos, también demostraron la ausencia de correlación entre las presiones auriculares contra el volumen latido y trabajo ventricular en modelos con aumento de la presión intratorácica (y otras presiones yuxtacardiacas), reportaron que aunque la $\mathrm{P}_{\mathrm{RA}}$ incrementaba, el volumen latido caía ${ }^{26,35}$. La forma en la que Sarnoff presentó sus curvas es la que actualmente se conoce como "curva de respuesta a volumen" o de Frank-Starling, pues previamente Starling había reportado que la $P_{R A}$ aumentaba hasta alcanzar el límite de la capacidad de adaptación cardiaca al volumen, desde ese punto la $\mathrm{P}_{\mathrm{RA}}$ aumentaba desproporcionalmente al volumen latido y el corazón se fatigaba. También realizó algunos experimentos interrumpiendo el flujo de salida de la aorta, descubriendo que la $\mathrm{P}_{\mathrm{RA}}$ podía aumentar $\sin$ incrementar el GC; para Starling la curva era solo una parte básica del desarrollo de la «ley del corazón», que señala que el trabajo miocárdico varía con la

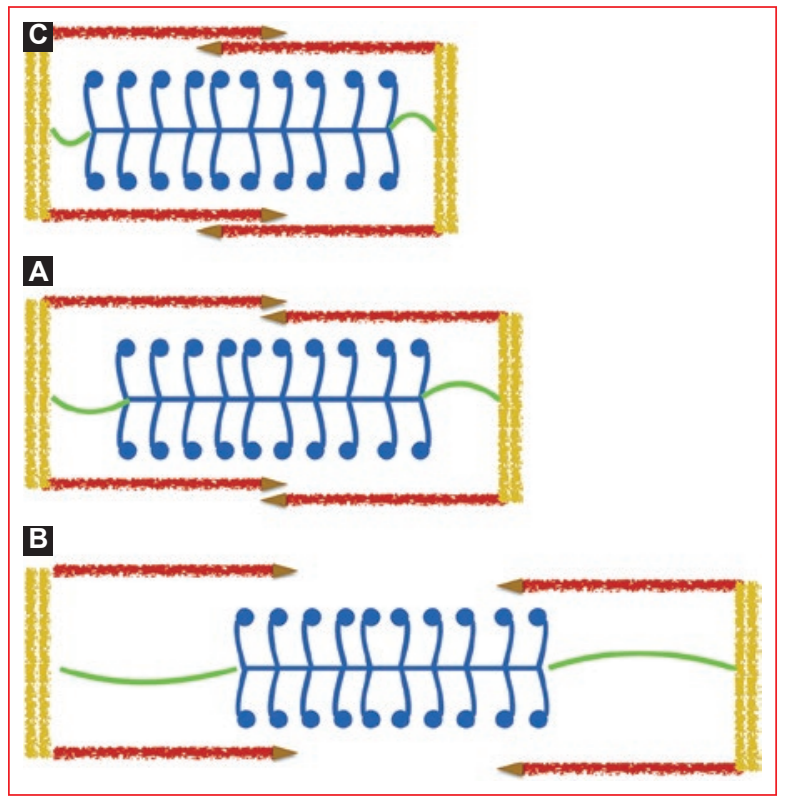

Figura 3. Diagrama de distensión de las fibras miocárdicas. A: muestra una sarcómera donde la miosina (azul) tiene adecuado contacto con la actina (rojo) para atraer a las bandas Z (amarillas) hacia el centro, óptima contracción de la fibra miocárdica, corresponde al punto A de la figura 1. B: muestra el efecto del aumento de la MCFP y la $P_{R A}$ sobre la miofibrilla, que distiende excesivamente la sarcómera, eso genera que la miosina sea incapaz de unirse adecuadamente a la actina, mientras que la titina (verde) se deforma y pierde capacidad contráctil, corresponde al punto $B$ de la figura 1. C: muestra una fibra medianamente distendida, donde la MCFP está disminuida y un porcentaje menor de miosina está libre para unirse a las bandas de actina y genera una contracción subóptima, que puede mejorar si se aumenta la distensión de esta, corresponde al punto $C$ de la figura 1 (elaboración de los autores basada en Guyton, et al., 200613).

MCFP: presión media de llenado circulatorio, mean circulatory filling pressure; $\mathrm{P}_{\mathrm{RA}}$ : presión de la aurícula derecha.

longitud inicial de la fibra del miocito ${ }^{32-34}$, es decir, que la capacidad inotrópica está estrechamente relacionada a la capacidad lusitrópica.

La fuerza de contracción (entiéndase inotropismo) en gran medida depende de qué tanto se distienda la fibra miocárdica. La capacidad contráctil del sarcómero está en función de la cantidad de cabezas de miosina que se unan a las bandas de actina para generar el acortamiento de la zona $\mathrm{H}$; cuando pocas moléculas de actina y miosina se logran aparear la contracción es menor, por lo que la distensión de la sarcómera expone a los receptores y promueve mejor acoplamiento entre 
actina y miosina, dicha distensión tiene un punto máximo, que se limita por la capacidad elástica de la proteína CapZ, actina y titina, ese punto máximo explica por qué las curvas de Starling y Sarnoff tienen un límite plano y no desciende cuando se agota la respuesta lusitrópica e inotrópica del mecanismo de Frank-Starling. La caída de esta curva representaría pérdida de la presión y volumen dentro de la cavidad, solo visible, hipotéticamente, en una ruptura ventricular (Fig. 3). Otro efecto de la banda de actina es que junto a esta también se elonga el receptor de troponina (molécula chaperona de la unión actina y miosina); al elongarse permite más ingreso de calcio y por lo tanto inhibición de este "chaperón", por consecuente, mejor apareamiento de actina y miosina, que resulta en más fuerza contráctil.

\section{Poscarga y su papel en la respuesta a volumen}

La poscarga es la fuerza que se opone al volumen latido, es decir, la fuerza que los ventrículos deben vencer para movilizar la sangre de forma anterógra$\mathrm{da}^{13,36}$ y ya que el sistema circulatorio tiene dos circuitos, los determinantes de la poscarga del ventrículo derecho (VD) no son iguales a los del ventrículo izquierdo (VI). La presión yuxtacardiaca nuevamente entra en discusión, pues si aumenta la poscarga del VI, disminuye el VR proveniente de la circulación pulmonar, esto aumenta la poscarga del VD, el abobamiento del VI puede aumentar la presión yuxtacardiaca y el volumen telesistólico del VD; si esto se perpetúa, aumentará la $\mathrm{P}_{\mathrm{RA}}$, lo que disminuye el VR y la respuesta a volumen $27-29,36,37$, un fenómeno semejante ocurre cuando se incrementa de forma súbita la poscarga del VD, por ejemplo en embolia pulmonar ${ }^{38}$, neumotórax a tensión o con aumento de la presión alveolar (léase presión positiva al final de la espiración elevada), maniobras de reclutamiento alveolar ${ }^{39}$. De forma inicial, a pesar de conservar la contractibilidad del VD, la MCFP y el VR normales, elevar la $P_{R A}$ tiene impacto negativo en la respuesta a volumen, donde el fenómeno de interdependencia ventricular afectará al VI, que no logrará vencer a su poscarga, y por lo tanto el circuito sistémico tendrá depleción del gasto y por consiguiente del volumen estresado, disminuyendo aún más el VR; en el infarto del VD la poscarga a nivel pulmonar se puede mantener en rangos normales, sin embargo, la contractibilidad del VD no es suficiente para vencerla y entonces aumenta la $P_{R A}$, con el impacto negativo que ya se ha mencionado previamente. La poscarga puede calcularse por varios métodos, como la elasticidad arterial dinámica y el acoplamiento ventrículo-arterial ${ }^{37,39-41}$, sin embargo, ese tema excede los alcances de este trabajo.

\section{Conclusiones}

La respuesta a volumen es la capacidad del miocardio de incrementar el GC en función del aumento del VR. Evaluar la respuesta a volumen es fundamental para prevenir las complicaciones de la sobrecarga de líquidos, sin embargo, no se logra la adecuada compresión sin un profundo entendimiento de la fisiología y los mecanismos por los cuales el sistema vascular y el corazón se adaptan o claudican ante la llegada de volumen. Debido a que los componentes del VR pueden ser analizados de forma independiente, su estudio permite al clínico optimizar cada uno de estos elementos en pro del paciente; esto es solo una fracción del complejo aparato circulatorio, cuando la respuesta a volumen falle, será momento de voltear a ver a la contractibilidad, la poscarga, el aporte, transporte y consumo de oxígeno, elementos interconectados y de cuyo balance depende la deseada homeostasis.

\section{Financiamiento}

El presente trabajo no ha recibido ninguna beca específica de agencias de los sectores público, comercial o sin ánimo de lucro.

\section{Conflicto de intereses}

Luis A. Gorordo-Delsol reporta recibir honorarios de Pfizer México SA de CV, Merck Sharp \& Dohme Comercializadora $S$ de $R L$ de $C V$ y Abbott Laboratories de México SA de CV por actividades académicas no relacionadas con el presente trabajo. También es miembro del comité editorial de la Revista de Educación e Investigación en Emergencias, sin participar en el proceso de dictamen del manuscrito.

Los otros autores declaran no tener conflictos de interés.

\section{Responsabilidades éticas}

Protección de personas y animales. Los autores declaran que para esta investigación no se han realizado experimentos en seres humanos ni en animales. 
Confidencialidad de los datos. Los autores declaran que en este artículo no aparecen datos de pacientes.

Derecho a la privacidad y consentimiento informado. Los autores declaran que en este artículo no aparecen datos de pacientes.

\section{Bibliografía}

1. Merinos-Sánchez G, Gorordo-Delsol LA, Guamán-Crespo JO Hernández-López GD. Reanimación hídrica: tipos de líquidos y efectos adversos. Rev Educ Investig Emer. 2019;1(1):23-31.

2. Cherpanath TG, Aarts LP, Groeneveld JA, Geerts BF. Defining fluid responsiveness: a guide to patient-tailored volume titration. J Cardiothoracic Vasc Anesth. 2014;28(3):745-54.

3. Michard F, Teboul JL. Using heart-lung interactions to assess fluid responsiveness during mechanical ventilation. Crit Care. 2000;4:282-9

4. Saleh AS. Is the concept of fluid responsiveness evidence-based? Intensive Care Med. 2016;42(7):1187-8.

5. Marik PE, Monnet X, Teboul JL. Hemodynamic parameters to guide fluid therapy. Ann Intensive Care. 2011;1:1-9.

6. Marik PE. Fluid responsiveness and the six guiding principles of fluid resuscitation. Crit Care Med. 2016;44(10)1920-2.

7. Cecconi M, Hofer C, Teboul JL, Pettila V, Wilkman E, Molnar Z, et al. Fluid challenges in intensive care: the FENICE study: a global inception cohort study. Intensive Care Med. 2015;41(9):1529-37.

8. Marik PE. The physiology of volumen resuscitation. Curr Anesthesio Rep. 2014;4:353-9.

9. Monnet X, Rienzo M, Osman D, Anguel N, Richard C, Pinsky MR, et al Passive leg raising predicts fluid responsiveness in the critically ill. Crit Care Med. 2006;34:1402-7.

10. Weil MH, Henning RJ. New concepts in the diagnosis and fluid treatment of circulatory shock. Anesth Analg. 1978;58(2):124-32.

11. Vincent JL, Wel MH. Fluid challenge revisited. Crit Care Med. 2006; 34:1333-7.

12. Shippy CR, Appel PL, Shoemaker WC. Reliability of clinical monitoring to assess blood volumen in critically ill patients. Crit Care Med. 1984;12:107-12.

13. Guyton AC, Hall JE. Textbook of medical physiology. $11^{\text {th }}$ ed. Pennsylvania, EE.UU.: Elsevier-Saunders; 2006.

14. Baker RJ, Kozoll DD, Mayer KA. The use of surface área as a basis for stablishing normal blood volumen. Surg Gynecol Obstet. 1957;104:185.

15. Aldrete JA, Franco-Gutiérrez LM. Análisis de métodos de estimación de volumen sanguíneo circulante. Rev Mex Anest. 1987;10:155-7.

16. Magder S. Volume and its relationship to cardiac output and venous return. Crit Care. 2016;20:217:1-11.

17. Deschamps A, Magder S. Effects of heat stress on vascular capacitance. Am J Physiol. 1994;266:H2122-9.

18. Magder S. Role of venosu elastic recoil pressure as a determinant of steady-state cardiac output. J Appl Physiol. 2009;107:370.
19. Rothe CF. Reflex control of veins and vascular capacitance. Physiology Rev. 1983;63(4):1281-95

20. Beard DA, Feigl EO. Understanding Guyton's venous return curves. Am J Physiol Heart Circ Physiol. 2011;301(3):H629-H633.

21. Weber EH. On the apllication of wave theory to the theory of the circulation of the blood and in particular to the pulse. Arch Anat Physiol. 1851;18:497-501.

22. Henderson WR, Griesdale DEG, Walley KR, Shell AW Clinical review: Guyton - the role of mean circulatory filling pressure and right atrial pressure in controlling cardiac output. Crit Care. 2010;14(243):1-6.

23. Brengelmann GL. A critical analysis of the view that right atrial pressure determines venous return. J Appl Physiol. 2003;94:849-59.

24. Pinsky MR. The classical Guyton view that mean systemic pressure, right atrial pressure, and venous resistance govern venous return is/is not correct. J Appl Physiol. 2006;101:1528-30.

25. Guyton AC. Determination of cardiac output by equating venous return curves with cardiac response curves. Physiol Rev. 1955;35(1):123-29.

26. Sarnoff SJ. Myocardial contractility as described by ventricular function curves. Physiol Rev. 1955;35:107-22.

27. Levy MN. The cardiac and vascular factors that determine systemic blood flow. Circ Res. 1979;44:739-47.

28. Bendjelid K. Right atrial pressure: determinant or result of change in venous return? Chest. 2005;128(5):3639-40

29. Gelman S. Venous function and central venous pressure: a physiologic story. Anesthesiology. 2008;108:735-48.

30. Magder S. More respecto for the CVP. Intensive Care Med. 1998;24:651-3.

31. Permutt S, Riley S. Hemodynamics of collapsible vessels with tono: the vascular waterfall. J Appl Physiol. 1963;18(5):924-32.

32. Berlin DA, Bakker A. Starling curves and central venous pressure. Crit Care. 2015;19(55):1-7.

33. Buckberg G, Mahajan A, Saleh S, Hoffman JIE, Coghlan C. Structure and function relationship of the helical ventricular myocardial band. J Thorac Cardiovasc Surg. 2008;136:578-89.

34. Katz AM. Influence of altered inotropy and lusitropy on ventricular pressure-volume loops. J Am Cardiol College. 1988;11(2):438-45.

35. Sarnoff JS, Berlund E. Ventricular function: I. Starling's law of the heart studied by means of simultaneous right and left ventricular function curves in the dog. Circulation. 1954:9:706-18.

36. Vincent JL. Undestanding cardiac output. Crit Care. 2008;12(4):174

37. Ochagavía A, Zapata L, Carrillo A, Rodríguez A, Guerrero M, Ayuela JM. Evaluación de la contractilidad y la poscarga en la unidad de cuidados intensivos. Med Intensiva. 2012;36:365-74.

38. Gorordo-Delsol LA, Zamora-Gómez SE, Hernández-López GD, García-Román MTA, Jiménez-Ruiz A, et al. Tromboembolia pulmonar: revisión sistemática y algoritmo diagnóstico-terapéutico. Rev Hosp Jua Méx. 2015;82(2):105-13.

39. Pinsky MR. The right ventricle: interaction with the pulmonary circulation. Crit Care. 2016;20:266

40. Subramaniam B, Talmor D. Echocardiography for management of hypotension in the intensive care unit. Crit Care Med. 2007;35:S401-S407.

41. Kubba S, Davila CD, Forfia PR. Methods for evaluationg right ventricular function and ventricular-arerial coupling. Prog Cardiovasc Dis. 2016;59(1):42-51. 